\title{
40. Radio Astronomy (Radioastronomie)
}

\author{
PRESIDENT: M. Morimoto (Japan) \\ VICE-PRESIDENT: J.B. Whiteoak (Australia) \\ ORGANIZING COMMITTEE: D.C. Backer (USA), R. Fanti (Italy), \\ R. Guesten (Germany), J. Moran (USA), J.M. van der Hulst (Netherlands), \\ R. Davis (UK), J. Gomez-Gonzales (Spain), F.R. Colomb (Argentina), \\ Ye Shuhua (China), P.G. Mezger (Germany), E. Gerard (France), \\ T. Velusamy (India), L.B. Baath (Sweden), E.E. Baart (S. Africa), \\ I. Gossachinskiy (CIS)
}

\section{INTRODUCTION}

Radio astronomy has been providing new frontiers to astronomy and this was still true during the last three years. In this report, we concentrate on discussing such new possibilities. Observational results and interpretations are discussed more in detail in the reports of other commissions and we will avoid mentioning these except when the results demonstrate that a new possibility is opening up.

\section{RADIO TELESCOPES}

\subsection{Single Aperture Telescopes}

\subsubsection{Sub-millimeter wavelength telescopes}

Sub-millimeter wave astronomy is growing very fast. Another major observing facility has been added to the three already existing.

The Caltech Submillimeter Observatory (CSO) telescope is operating routinely at 230, 345 and $460 \mathrm{GHz}$. A $5 \times 5$ element array receiver is planned for $490 \mathrm{GHz}$.

For the 15-m James Clerk Maxwell Telescope (JCMT) on Mauna Kea a 64-element array heterodyne receiver at $345 \mathrm{GHz}$ is being developed at Cambridge University (MARS). For continuum observations, 91-pixel and 37-pixel bolometer arrays are being built for operation at 650 and $350 \mathrm{GHz}$ respectively (SCUBA).

For the Swedish-ESO Submillimeter Telescope (SEST) a new SIS receiver covering the band 320-360 GHz was installed in 1992 (Nyman et al., 1990, Liege International Astrophysical Colloquium, p. 159). Its noise temperature is $200 \mathrm{~K}$ (SSB) and system temperatures as low as $420 \mathrm{~K}$ (SSB) have been realized

The first permanent sub-millimeter wavelength telescope for location at the South Pole, the 1.7-m Antarctic Submillimeter Telescope and Remote Observatory (AST/RO) instrument is fully assembled and being tested with a $490 \mathrm{GHz}$ receiver in Boston before deployment in 1994 (Lo et al., 1991, IAU Symposium No. 148, p.112). Its first major task will be a CI line survey.

The Sub-millimeter Telescope (SMT), a joint project of the Max Planck Institut für Radioastronomie (MPIfRA) and the University of Arizona, was dedicated in September 1993 (Baars et al., 1990, Liege International Astrophysical Colloquium, p. 293). The spectroscopic systems have SIS receivers at 350,450 , and $690 \mathrm{GHz}$ and acousto-optical spectrometers. He-cooled bolometers are available for continuum observations.

A $30-\mathrm{cm}$ rocket borne telescope with a 12 -element bolometer array is under construction in Japan for launch in 1994. It will observe dust emission in the Orion region at wavelengths of 0.5 and $0.25 \mathrm{~mm}$. 


\subsubsection{Large millimeter to meter wavelength telescopes}

A 100-m telescope (Green Bank Telescope; GBT) is under construction in Green Bank, USA by National Radio Astronomy Observatory (NRAO) as a replacement of the old 91-m transit telescope. It will use offset Gregorian optics to reduce diffraction caused by the subreflector and its support structure. This will greatly improve aperture efficiency and reduce side lobe level and noise temperature. It will be equipped with a laser survey system for actively controlling the surface, and will have a useful performance at $\mathrm{mm}$-wavelengths. Its completion is planned for 1995 and it will be a very important addition to radio astronomy.

The 14-m telescope of the Five College Radio Astronomy Observatory (FCRAO) has been used extensively with a 15 element focal plane array (QUARRY), which operates in the 86$115 \mathrm{GHz}$ band (Goldsmith et al., 1991, Bull. AAS, 23, 1452). Efficient signal processing is provided by a new $15 \times 1025$ channel spectrometer.

The NRAO 12-m telescope has an SIS receiver at $100 \mathrm{GHz}$ that has a receiver temperature of $19 \mathrm{~K}$ (DSB) and system temperatures as low as $130 \mathrm{~K}$ (SSB). The Schottky diodes in the 8 element $(4 \times 2)$ array receiver for the $215-245 \mathrm{GHz}$ band have been replaced by SIS junctions with fixed tuning.

The surface of the 45-m telescope of the Nobeyama Radio Observatory (NRO) was reset in 1992, further improving its rms surface accuracy from 95 to 65 microns. It is now equipped with a 4-element SIS receiver at $115 \mathrm{GHz}$ and a 7 -element bolometer array at $150 \mathrm{GHz}$. A 25-element SIS array receiver is under development.

The surface accuracy of the 100-m telescope of MPIfRA and the 37-m telescope of Haystack Observatory were improved to have useful performance up to $100 \mathrm{GHz}$. The improvements will be of particular importance for mm-wave VLBI.

The size of the panels of RATAN-600 has been increased from $7.4 \mathrm{~m}$ to $11.4 \mathrm{~m}$ and the geometrical area in the zenith is about $15,000 \mathrm{~m}^{2}$. Spillover was decreased to less than $3 \mathrm{~K}$. FET and HEMT receivers give sensitivities better than $10 \mathrm{mK}$ for $1 \mathrm{sec}$ integration at $\mathrm{cm}$ wavelengths. Part of its surface accuracy was improved to $0.085 \mathrm{~mm}(\mathrm{rms})$ and a test for quasi-real time adaptive optics using simple one dimensional holography was successful.

The 54-m "Arecibo" style antenna of the Radio Measurements Institute in Armenia is situated in the Aragats mountains. The rms surface accuracy is 83 microns and operation up to $150 \mathrm{GHz}$ is possible. The effective use of the facility has been hampered by the lack of receivers and by operational constraints.

The FCRAO in collaboration with several groups in Mexico is planning a 50-m, radome enclosed, telescope that will operate in the range of 80 to $350 \mathrm{GHz}$. The preliminary design consists of a parabolic primary reflector of 126 hexagonal panels that are actively controlled. A new mirror system for correcting spherical aberration is being installed on the Arecibo 310-m telescope. Together with recent resurfacing and a new shielding fence, it will greatly enhance the performance of this powerful telescope. This will be important for planetary radar, pulsar timing and many other types of observations.

Constructions of medium sized $(25-30 \mathrm{~m})$ telescopes in Italy, China, Poland, and Russia are in progress. They will be used mainly for VLBI observations.

\subsubsection{Survey telescopes in sub-millimeter and millimeter-wavelength astronomy}

The Cologne 3-m telescope on Gornergrat, Switzerland, has a surface accuracy of 30 microns and receivers at 115 and $230 \mathrm{GHz}$. It is equipped with acousto-optical spectrometers (Tolls et al., 1990, Liege International Astrophysical Colloquium, p. 299).

The 0.9-m telescope of the Harvard-Smithsonian Center for Astrophysics in Cambridge (formerly at Columbia University) has been used during the past triennium to map molecular clouds in the outer Galaxy and M31 in the CO 1-0 line (Koper et al., 1991, Ap. J., 383, L11) and to search for gas far out of the plane in our Galaxy.

The 0.6-m telescope of the University of Tokyo and Nobeyama Radio Observatory, located at Nobeyama, has been dedicated to mapping the Galactic plane in the CO 2-1 line for comparison with the Columbia-CFA survey.

The pair of 4-m telescopes at the Nagoya University, Japan, is involved in a 600,000-point survey of the CO 1-0 line covering 1,500 square degrees of sky.

There is a plan in Japan to build a 2-m sub-mm telescope on the summit of the Mount Fuji. 


\subsubsection{Multi-beaming of radio telescopes}

If more than one feed horn are arranged at the focus, the telescope will have more than one beam on the sky. This will ease the long-standing frustration of radio astronomers who would like to observe several positions on the sky simultaneously.

The main aims of multi-beaming are, a) to greatly reduce the time necessary to survey a certain area of sky, and b) to remove the effects of atmospheric fluctuations by comparison of adjacent beams. These two points are extremely important in mm-wave telescopes, where observations are severely limited by fluctuations in atmospheric emission and absorption, and where the telescope beams are usually very narrow.

For such arrangements to be effective, there will occur new technical problems. At present, the most important are the signal and data handling and feed designs for effective use of the focal plane. Developments of wide-angle optical system to get a focal plane like optical telescopes will become very important. After all such developments, eventually, all radio telescopes will have fields of view. Then new possibilities will open up.

Among some obvious examples of possibilities with multi-beaming are phase referenced VLBI observations of sources nearby in the sky, online correction of telescope pointing using a point source in the field, correcting and/or adjusting the telescope beam by coherently combining beam outputs, and so on.

The first attempt at multi-beaming was with the old $91-\mathrm{m}$ transit telescope of NRAO in the early 60's. It is also extensively used with the $100-\mathrm{m}$ telescope of MPIfRA.

At present, efforts are more concentrated on $\mathrm{mm}$-wavelength operation. The first one was the 8-beam arrangement on the 12-m mm-wave telescope of NRAO. It used 8-feed horns with Schottky mixer receivers. Because of the limitation of receiver performance this system was not used extensively and is being replaced by SIS receivers.

At present, 15-beam arrangement on the 14-m telescope of FCRAO, an 8-beam system of the $12-\mathrm{m}$ telescope of NRAO and 4-beam system on the 45-m telescope of Nobeyama Radio Observatory are in use. There are many more such systems being planned or about to be installed on telescopes. They are listed in Table 1.

Table 1. Multi-Beam Receivers

\begin{tabular}{clcll}
\hline Institute & Frequency & Beams & Type & Comment \\
\hline NRO & $108-116 \mathrm{GHz}$ & 4 & SIS & fixed tuning \\
& $80-116 \mathrm{GHz}$ & 25 & SIS & fixed tuning * \\
& $40-50 \mathrm{GHz}$ & 6 & SIS & planned \\
& $1,2 \mathrm{~mm}$ & 7 & bolometer & \\
MPIfRA & $32 \mathrm{GHz}$ & 9 & HEMT & $*$ \\
& $15 \mathrm{GHz}$ & 4 & HEMT & $*$ \\
& $10.55 \mathrm{GHz}$ & 4 & HEMT & \\
IRAM & $4.75 \mathrm{GHz}$ & 2 & HEMT & $*$ \\
& $230,850 \mathrm{GHz}$ & 7 & bolometer & \\
FCRAO & $80-116 \mathrm{GHz}$ & 15 & SIS & planned \\
NRAO & $215-245 \mathrm{GHz}$ & 8 & SIS & fixed tuning * \\
JCMT & $0.35,0.6 \mathrm{~mm}$ & 91 & bolometer & $*$ \\
& $0.75,1.1,1.4,2 \mathrm{~mm}$ & 37 & bolometer & $*$ \\
\hline
\end{tabular}

The 64-element array receiver being developed at Cambridge for the JCMT will coherently combine the outputs so that beam correction etc. is possible.

With the RATAN-600, a new "array mode" is being designed to have a field of view up to the beam of single panel. 


\subsection{Antenna Arrays}

In Table 2, parameters of Arrays/Interferometers are summarized.

\subsubsection{Sub-millimeter wavelength arrays}

The Smithsonian Submillimeter Array (SMA) is midway through construction and is expected to be completed by 1997 . The initial instrument consists of six 6-m antennas distributed among 24 pads in 4 concentric layouts. The antennas will be placed along the sides of Reuleaux triangles, which have been found to provide the most uniform uv plane coverage within a circular boundary in the uv plane.

The array will be sited on Mauna Kea near the CSO and JCMT, and will be linked for combined operation.

The JCMT and CSO antennas have been linked to form the first sub millimeter wavelength interferometer. The baseline is $\mathbf{1 5 0}$ meters. Even with only a single baseline this instrument will be able to measure the angular sizes of compact objects, act as a spatial filter to study absorption lines against compact background objects and measure the relative positions of maser clusters. Initial results include the mapping of water vapor masers in the stellar envelop of VY CMa (Carlstrom, 1993, Bull. $A A S, 25,859$ ).

\subsubsection{Millimeter wavelength arrays}

The Berkeley Illinois Millimeter Array (BIMA) is in the process of being upgraded from 3 to 9 antennas. Two additional antennas have been funded and will be built within two years. Long baseline stations have been added, increasing the maximum baseline to 800 meters. Presently the array operates in the $85-115 \mathrm{GHz}$ band but three more receivers will be added per antenna to cover the $70-90 \mathrm{GHz}, 130-180 \mathrm{GHz}$, and $210-270 \mathrm{GHz}$ bands. The new correlation spectrometer, based on the Bos chip, can handle a bandwidth of $800 \mathrm{MHz}$ in a variety of modes with up to four separate spectra and up to 1024 channels/baseline.

The IRAM array on the Plateau de Bure was expanded to 4 antennas in 1993. The surface accuracy of the fourth $15-\mathrm{m}$ antenna is 70 microns. Funds have been approved for 2 more antennas. The surfaces of these antennas will be made from machined aluminum rather than aluminum-coated carbon fiber.

The millimeter array of the Nobeyama Radio Observatory (NRO) will be expanded from five to six antennas. The new antenna is expected to have rms surface accuracy of $\mathbf{4 0}$ microns and $78 \%$ aperture efficiency at $115 \mathrm{GHz}$ (compared with 60 microns on the older antennas). A new correlator is under construction based on an LSI chip with 32 lags operating at 32 $\mathrm{MHz}$ clock rate. With time demultiplexed operation it will handle a $2 \mathrm{GHz}$ bandwidth with 256 channels/baseline. The $45-\mathrm{m}$ telescope will be added to the array, greatly enhancing its sensitivity.

NRO has begun to plan for a next generation millimeter wavelength array of approximately fifty $10-\mathrm{m}$ antennas. Sites under consideration are in Hawaii, Tibet and northern Chile. Extensive site testing in northern Chile with phase monitoring instrumentation will begin soon.

A four year development plan for a Millimeter Array (MMA) has been submitted by NRAO to the NSF. The array would have forty 8-meter elements. Testing of the primary sites on South Baldy, New Mexico, and Mauna Kea, Hawaii, continue. The antenna design is in an advanced state and now consists of an uncounterweighted slant-axis parabola.

\subsubsection{Centimeter to meter wavelength arrays}

MERLIN was up-graded with the addition of the 32-m telescope at Cambridge. Now it has 7 telescopes and 21 baselines. Maximum baseline is now $218 \mathrm{~km}$. Correlator was improved to have $2 \times 16 \mathrm{MHz}$ channels with 2 bit quantization. With a 12-hour integration, its sensitivity is $1,000 \mathrm{~K}$ in brightness and angular resolution is 50 mas at $5 \mathrm{GHz}$.

The Giant Meter-wavelength Radio Telescope (GMRT) with thirty 45-m telescopes is under construction in India. It will work between $40 \mathrm{MHz}$ and $1.4 \mathrm{GHz}$. Two of the antennas are now complete. 
The Nobeyama Radio Heliograph was completed in 1992. It is a T-array of eighty four 80$\mathrm{cm}$ antennas on the baselines of $500 \mathrm{~m} \mathrm{EW}$ and $200 \mathrm{~m}$ NS. It produces 10 " resolution maps of the Sun with a maximum time resolution of $50 \mathrm{~ms}$. At present, the observing frequency is $17 \mathrm{GHz}$ and an addition of a second frequency of $34 \mathrm{GHz}$ is planned (also see Section 5.3).

For observing anisotropy of the Cosmic background radiation, an interferometer consisting of three $70-\mathrm{cm}$ telescopes is being constructed at Cambridge.

The Compact Array of the ATNF now provides dual polarization, dual frequency operation in four frequency bands between $20 \mathrm{~cm}$ and $3.5 \mathrm{~cm}$, on all of its $22-\mathrm{m}$ antennas.

\section{VLBI}

\subsection{Networks}

The Very Long Baseline Array (VLBA) is a dedicated VLBI telescope, consisting of ten 25 $m$ antennas distributed over the territory of USA, equipped with "state of the art" receiving, recording, correlation and image processing facilities. It will provide imaging observations with up to 0.1 -mas resolution depending on observing frequencies $(0.3$ through $40 \mathrm{GHz})$.

The Joint Institute for VLBI in Europe (JIVE) is being established as the permanent basis for VLBI observations in Europe. The Institute is sited in the Netherlands and its first major project is to construct a new large correlator, capable of handling data from 16 stations. The Institute will also provide for processing space VLBI data. Planned completion is in 1997. This, together with the existing corrector in Bonn, will ease the bottleneck in processing.

VLBI networks on smaller scales are being established or planned.

A Chinese network consists of two 25-m telescopes, one in Shanghai and the second one in Urumqi (3,000 km north-west of Shanghai). Participation of existing telescopes in China and addition of new telescopes are being considered.

The Australia Telescope (AT) is being equipped with S2 type recording terminals and has telescopes at Narrabri, Coonabarabran and Parkes. These are used in conjunction with other antennas around Australia and overseas. This network will play a very important role especially for observing southern hemisphere sources, and in space VLBI.

KNIFE is a link between the Kashima 34-m and Nobeyama $45-\mathrm{m}$ telescopes in Japan, mainly devoted to $\mathrm{mm}$-wave observations. There is a plan to add more telescopes.

QUASAR is a project to distribute four 32-m telescopes in the former Soviet Union, mainly devoted to geodetic observations. Two antennas and associated equipment are being constructed and will be in operation soon.

The Italian Network now has two 32-m antennas and actively participates in EVN observations.

\subsection{Millimeter-Wave VLBI}

Mm-VLBI can provide angular resolution of up to 20 microarcsec using baselines on Earth. It can penetrate the dense plasma and see the cores of active galactic nuclei (AGN). The determination of precise positions of stellar maser emission will be also very important.

The first successful mapping with global baselines was reported in the late 80's (Bartel et al. 1988, Nature, 334, 131). After intense efforts to overcome technical difficulties, increasing numbers of maps with 50-100-microarcsec resolution are being obtained at frequencies between 40 and $100 \mathrm{GHz}$. EVN and VLBA have included $40 \mathrm{GHz}$ in their observing frequencies. Attempts to detect fringes on global baselines at $230 \mathrm{GHz}$ are being made without overwhelming success.

Further improvements in mm-wave and digital technologies, increasing participation of large telescopes (especially up-graded 100-m telescope of MPIfRA and 37-m telescope of Haystack Observatory) and increasing enthusiasm will make mm-wave VLBI a very important topic in the coming years.

\subsection{Space VLBI}

The first successful experiment using a tracking and data relay satellite (TDRSS) as the space element demonstrated the technical feasibility and scientific potential of space VLBI. Two projects are in progress, both with a planned launch in 1996. One is RADIOASTRON 
using a Russian satellite and the other is VSOP (VLBI Space Observatory Program), using a Japanese satellite. Table 3 lists the parameters. The former has an elongated orbit which will be suitable for measuring source sizes and brightness temperatures, which are of fundamental importance to cosmology and the nature of cores of AGNs. The latter has a lower orbit and will give high quality images of AGNs

Both missions require very complicated and large scale international cooperation. Close cooperation is necessary in policy decisions, general and detailed planning, supply of dispensable items and so on. They will not only be exciting astronomical projects, but also interesting experiments in the sociology of science.

Table 3. Space VLBI Projects

\begin{tabular}{lrr}
\hline Parameters & RADIOASTRON & \multicolumn{1}{c}{ VSOP } \\
\hline Orbit & & \\
$\quad$ Period & $28 \mathrm{~h}$ & $6 \mathrm{~h}$ \\
Inclination & $51.6 \mathrm{deg}$. & $31 \mathrm{deg}$. \\
Apogee Altitude & $41,000 \mathrm{~km}$ & $20,000 \mathrm{~km}$ \\
Perigee Altitude & $2,000-4,000 \mathrm{~km}$ & $1,000 \mathrm{~km}$ \\
Telescope Diameter & $10 \mathrm{~m}$ & $8 \mathrm{~m}$ \\
Receivers & $0.327,1.6,5,22 \mathrm{GHz}$ & $1.6,5,22 \mathrm{GHz}$ \\
Video Bandwidth & $2,4,8 \mathrm{MHz}$ & $16,32 \mathrm{MHz}$ \\
Video Channels & $1,2,4$ & 1,2 \\
Total Data Rate & $32,64,128 \mathrm{Mbps}$ & $128 \mathrm{Mbps}$ \\
\hline
\end{tabular}

\subsection{Recording Systems}

There are 3 different recording systems based on different tapes and tape recorders. They are the Mk IIIIa or VLBA system using lateral recording instrumentation recorders and developed in USA, the K4 system using helical scan data recorders and developed in Japan, and the S2 system using commercial video recorders developed in Canada.

They are not compatible with each other and this causes complications in the VLBI community. At present, a compromise is being sought in which tape copiers will enable transfers between the systems. Efforts to make clear definition and machine independent interface (Generic Interface) have been done.

The sensitivity of VLBI observations depends critically on bandwidth, which is limited by recording speed. This limitation has more serious effects on $\mathrm{mm}$-wave observations, where long integration is not possible because of phase fluctuations in the terrestrial atmosphere.

The Mk IV system developed in Haystack can take with a data rate of $1 \mathrm{Gbps}$ with a factor of 4 improvement in speed compared to the Mk IIIa.

A burst sampling method is being developed in Japan. In this method data are temporarily stored in a fast semiconductor memory and recorded on tape at a reduced speed. A pilot model having a $4 \mathrm{Gbps}$ instantaneous data rate for $8 \mathrm{sec}$ (roughly, the coherence time at 40 $\mathrm{GHz}$ ) is being developed at Nobeyama Radio Observatory.

\section{MM AND SUB-MM WAVE ASTRONOMY}

Development of sub-mm wavelength astronomy is very fast. There are already 4 major facilities, of which one is in the southern hemisphere, and construction of an array telescope is in progress (see Section 2.1).

Observations of molecular and dust emission in star forming regions in and outside the Galaxy will become more active. 
Molecular line observations form a major branch of $\mathrm{mm}$ and sub-mm wave astronomy. The discovery of high-redshift CO has stimulated a new area of extragalactic study (see Section 7).

Mm-wave VLBI is becoming a major field of research as it offers the highest angular resolution presently possible.

Improvements in SIS receivers still continue to lower noise temperatures and higher frequencies. (overview by Blundell and Tong, 1992, Proc. IEEE, 80, 1702, Special Issue on Terahertz Technology).

\section{RADIO ASTROMETRY}

\subsection{Reference System}

Connected-element arrays and VLBI networks are now capable of determining source positions much more accurately than by optical means. The present accuracy is 10 mas for the reference system, but tens of microarcsec for relative positions between sources close to each other.

The lack of southern reference sources has degraded the accuracy of the reference system in the southern sky. The situation is being improved by VLBI observations from observatories in southern hemisphere.

It has not been easy to connect this new reference system to the old optical reference system, because the optical system is based on positions of stars, while the radio system is based on extragalactic sources.

The sensitivity of VLBI and MERLIN has been improved to make observations of radio stars possible. The accuracy of positions from such observations are sufficient to link radio and optical reference systems.

\subsection{Dynamics of the Galaxy and galaxies}

The improved MERLIN can measure positions of radio stars and pulsars to an accuracy of a few mas.

Using three 70-m telescopes of Deep Space Network (DSN), the positions of 11 stars relative to nearby extragalactic sources were determined using a technique of phase-referenced VLBI (Lestrade et al., 1992, A. Ap., 258, 112). The precision in relative position and parallax was 0.1-0.7 mas, and in proper motion was 0.04-0.4 mas/year. In the case of $\mathrm{CrB}$, with the reference source only 0.5 degrees away and with 12 VLBI observations, parallax was determined to be $43.99 \pm 0.07$ mas (Lestrade et al., 1992, $A$. Ap., 258, 112). This must be the best known distance outside of the solar system.

The improvements of telescope sensitivity will make it possible to find a reference source closer in the sky than before. Multi-beaming of telescopes will make it possible to record simultaneously the reference source and the source of interest. There is a proposal to build two identical antenna clusters for phase referenced VLBI. Use of these new technique will improve the accuracy of position measurements to better than 10 microarcsec.

With this accuracy, proper motions and parallaxes of various objects in the Galaxy can be fairly accurately determined, and the dynamics inside the Galaxy studied more extensively.

Accurate timing measurements of pulsars also provide high accuracy in position and in some cases accelerations.

For 10-microarcsec accuracy in position measurements obtained ten years apart, it would be possible to detect a motion of $250 \mathrm{~km} / \mathrm{s}$ for an object at a distance of $10 \mathrm{Mpc}$.

Thus radio astrometry in the near future will bring major impacts on dynamics of the Galaxy and galaxies.

\subsection{Solar Radio Astronomy}

For the Nobeyama Radio Heliograph, described in 2.2.3, simultaneous observations with Solar X-ray imaging satellite "Yohkoh" of active regions and flares are producing important results. 
The 14-m telescope of Itapetinga, Brazil is fitted with a 5-horn receiving system at $22 \mathrm{GHz}$. With overlapping beam, this system can measure positions of solar bursts with a time resolution of milliseconds.

VLA and other arrays are also used to observe active regions and sometimes flares in microwave frequencies.

The Nançay Radio Heliograph is now producing interesting two-dimensional pictures of the sun at 150 and $450 \mathrm{MHz}$.

\subsection{Radio Stars}

The high sensitivity of VLA enables radio emission from stars to be readily detectable. The recent upgrade of MERLIN has provided the sensitivity to detect a star with radio brightness of $1,000 \mathrm{~K}$ and resolution of $50 \mathrm{a} . \mathrm{u}$. at a distance of $1 \mathrm{kpc}$. It can detect and resolve the photospheric thermal emission of many late-type stars, and thermal and non-thermal emission associated with stellar winds of early-type stars etc. Improvements in sensitivity in VLBI observations will enable stars to be detected and resolved.

Stellar maser sources are becoming popular subjects for VLBI observations. Following the pioneering works of University of Massachusetts and Haystack (Rosen et al., 1978, Ap. J., 222, 132; Spencer et al., 1979, Ap. J., 230, 449), observations are being made with KNIFE, VLBA and EVN. Astrometric observations have revealed that the maser spots are near the star rather than in the exosphere.

The impact of radio star observations on the astrophysics of stars, especially on mass loss processes, will be very significant.

\section{PULSARS}

Pulsars, even after 25 years since their discovery, are still prime objects for studies of the nature and evolution of compact objects, testing various gravitational theories, interstellar scintillation, gravitational field and other properties of globular clusters, and so on.

Eight millisecond pulsars were discovered in the galactic disk, being roughly double the number known there. Outstanding among them is the very strong ( $1 \mathrm{Jy}$ at $400 \mathrm{MHz}$ ) PSR J0437-4713 (Johnston et al., 1993, Nature, 361, 613), which is binary in a 5.7 day orbit with a white dwalf companion (Bell et al., 1993, Nature, 364, 603). It is the nearest and brightest millisecond pulsar known. Pulsed gamma-ray emission was detected by the Compton Gamma Ray Observatory for PSR B1706-44 (Thompson et al., 1993, Nature, 359, 615) and PSR B 1055-52 (Fierro et al., 1993, Ap. J., 413, L27).

The success of pulsar research is due to the continuous supply of new varieties of pulsars through discoveries in new surveys. The detection of millisecond pulsars has had a strong impact due to the high stability of the pulse rates (Taylor Jr., 1991, Proc. IEEE, 79, 1054). The efficiency of surveys depends on the volume and resolution in a multi-dimensional parameter space, and requires handling a large amount of data and fast computations. For example, the Parkes pulsar processor samples every 0.3 milliseconds and has 256 frequency channels (Johnston et al., 1992, Mon. Not. R. astr. Soc., 255, 401). In some cases, searches are made not only for pulse rates and dispersion, but also acceleration of the rate.

Especially for millisecond pulsars, the effect of smearing of pulses due to dispersion is severe, and improvements in search techniques are required. There is continual progress in digital and computational technologies, and this will dictate the progress of pulsar research.

Of course, the performance of telescopes is as important as the data processing. The role of large telescopes is very important. The upgrade of the Arecibo telescope, completion of GMRT and GBT will add more power in the searching and studying of pulsars.

Extensive use of multi-beam arrangements in these large telescopes will also accelerate discoveries of new pulsars.

The improved performance of the MPIfRA $100-\mathrm{m}$ has resulted in the detection of a pulsar at $36 \mathrm{GHz}$ (Wielebinski et al., 1993, A. Ap., 272, L13).

With the detection of many more pulsars, especially with millisecond or sub-millisecond periods, the improved accuracies in timing observations, and the longer times over which observations are made, will provide opportunities for new areas of study such as a new time standard, a new distance scale, dynamics of the Galaxy, globular clusters, pulsar-blackhole pairs and so on. The season of discoveries will continue. 


\section{MOLECULES AND STAR FORMING REGIONS}

Molecular line studies are still a major part of mm-wave astronomy.

New interstellar molecules continue to be detected. Molecules containing less-abundant atoms such as $\mathrm{P}, \mathrm{Na}, \mathrm{Cl}, \mathrm{Al}, \mathrm{K}$, and $\mathrm{Al}$ are on the list, and $\mathrm{Mg}$ was added recently.

Studies of proto-stellar disks will highlight new frontiers such as sub-mm wave observations. The determination of isotopic abundances will be very important.

A surprise detection of strong CO line emission with $z=2.3$ (see Section 9) will open up a new area of studies.

This rich field of discoveries now provides items for the reports of other commissions.

\section{AGN}

Improved angular resolution, image quality, sensitivity and larger numbers of samples result in a better understanding of core, jet and lobe configurations of AGNs.

However, even for mm-wave VLBI with global baselines, the "central engine" still cannot be proved, even for the nearest AGN's. We need space-space baselines or perhaps an entirely different approach.

\section{HIGH- $z$ GALAXIES}

Brown and Vanden Bout have detected $\mathrm{CO} J=3-2$ emission with $z=2.3$ in IRAS10214+4724 (1992, Ap. J., 397, L11). The CO luminosity is 20 times higher than the most gas-rich ultra-luminous infrared galaxies (Solomon et al., 1992, Nature, 356, 318). The intensity of such line emission does not vary as $D^{-2}$ but $(1+z)^{3} D^{-2}$, where $D$ is luminosity distance, and is easier to detect than previously thought. With further improvements in telescope sensitivity, many more detections will be made.

Then, there is no reason that other molecules do not to be detected. A new area of molecular and isotope chemistry is expected to open.

\section{PHENOMENA AND OBJECTS OF COSMOLOGICAL IMPORTANCE}

\subsection{Gravitational Lenses}

There are some 10 objects identified as gravitational lenses and more will be added. This has established that the gravitational lensing may be a rather widespread phenomenon.

There is one object, PKS1830-211, on which the detection of large intensity variations in two components is expected (Nair et al., 1993, Ap. J., 407, 46). This is a more direct evidence than just from the shape of the source, for the gravitational lens.

Determination of Hubble constant is possible from this time lag and geometry of the source. It gives a value between the present limits.

The motion of a lensed quasar is magnified by the gravitational lens. This will make measuring the motion of very distant object possible.

\subsection{Sunyaev-Zel'dovich Effect}

The Sunyaev-Zel'dovich (S-Z) effect is a decrease in cosmic microwave background radiation due to scattering by hot plasma in cluster of galaxies. Its successful detection was reported from single dish observations.

It can be used to probe intergalactic matter in the cluster of galaxies and also to estimate Hubble constant.

The first interferometric imaging observations using the Ryle Telescope (the upgraded 5-km telescope) have clearly shown the effect in a cluster, Abell 2218 (Jones et al., 1993, Nature, 365,320 ). It is much less susceptible to errors than previous observations due to confusing sources. The decrement of background brightness due to $S-Z$ effect was about $1 \mathrm{mK}$. This result combined with X-ray observations supports a lower value of Hubble constant. 


\subsection{Cosmic Microwave Background Radiation}

The Cosmic Background Explorer (COBE) clearly detected $10^{-5}$ fluctuations in background radiation on a scale size of 10 degrees in a frequency range of 30 and $90 \mathrm{GHz}$ (Smoot et al., 1992, Ap. J., 396, L1) This is the first firm detection of anisotropies. There have been several ground-based experiments sensitive to different scale sizes, and a few reported detections.

\subsection{Angular Size-z Relation}

Angular size of a standard 'rod' falls inversely proportionally to the distance $D$, for small distance. For $z$ approaching and exceeding unity, it falls off much more slowly than $D^{-1}$. It depends on the cosmological model, especially on deceleration parameter, $q_{0}$.

Many attempts have been unsuccessful because of uncertainties in the size of the rod etc. Using the size of the cores of AGNs, a clear indication of this "turn over" was seen (Kellermann, 1993, Nature, 361, 134). The result corresponds to $q_{0}=0.5$.

More confirming evidence is necessary, but it appears that we are about to see the curvature of the Universe.

\section{PROTECTION OF RADIO ASTRONOMY FREQUENCIES}

Over the last three years there has been some improvement in the protection of radio astronomy frequencies. The most noteworthy success has been for the frequency band 1610.6-1613.8 MHz, containing the $\mathrm{OH}$ transition at $1612.231 \mathrm{MHz}$, of particular importance in studies of $\mathrm{OH} / \mathrm{TR}$ objects. Over the last ten years, radio astronomy observations of this transition have been badly affected by satellite transmissions associated with the Russian GLONASS global navigation system. This system contains a number of satellites, each transmission using a $1-\mathrm{MHz}$ carrier and additional $10-\mathrm{MHz}$ band both entered at one of a series of frequencies separated by $0.5625 \mathrm{MHz}$ between 1602.56 and $1615.50 \mathrm{MHz}$. A milestone occurred at the World Radio Conference held by the International Telecommunication Union (ITU) in March 1992, when the status of radio astronomy in the $\mathrm{OH}$ band was increased from secondary to shared primary. This meant that transmitting services sharing the same frequency band (such as navigation services which include GLONASS) would be required to operate without causing harmful interference to radio astronomy observations. In November 1992, as a result of negotiations between the InterUnion Commission on the Allocations of Frequencies (IUCAF) and the Russian administration, a Joint experiment was undertaken in which a number of major observatories around the world made observations in the $\mathrm{OH}$ band while GLONASS transmitting frequencies were varied. In a summary of the observing results, it was recommended that GLONASS transmissions should be limited to center frequencies of $1608.75 \mathrm{MHz}$ and lower as a first urgent step, then subsequently to frequencies of $1605.375 \mathrm{MHz}$ and lower. It was further recommended that the GLONASS system should employ filtering to reduce out-ofband interference in both the $\mathrm{OH}$ bands at $1610.6-1613.8 \mathrm{MHz}$ and $1660-1670 \mathrm{MHz}$. In September 1993, the Russian administration announced that it would reduce the effects of GLONASS transmissions in the $\mathrm{OH}$ band, with an aim to removing all interference to radio astronomy observations by 1998 . Soon after this, observatories reported that GLONASS transmissions associated with six carriers in the $\mathrm{OH}$ band were no longer present.

There have been some activities in the area of international frequency protection, under control of the ITU. The International Radio Consultative Committee (CCIR), responsible for several Study Groups looking after the interests of various radiocommunication services (and including radio astronomy), was recently replaced by the Radiocommunication Bureau. The Study Group structure has remained unchanged. Study Group 7 has continued to seek improved protection for radio astronomy by producing appropriate documentation to be used as a basis for future changes to the radio regulations. A revised list of frequencies of the astrophysically most important spectral lines for radioastronomy, adopted at the 1991 IAU General Assembly as Resolution A2, has been included in a Study Group Recommendation. The Study Group is also producing documentation relative to interference to radio astronomy observations from satellite transmissions centered at frequencies outside radio astronomy 
bands, and to the protection of radio astronomy observations carried out from locations on the Moon which are shielded from emissions produced on Earth. A handbook is being produced which discusses radio astronomy in the context of an ITU radiocommunication 'service'. 


\section{References}

Reviews Relevant to this Report

Proceedings of IAU Symposia and Colloquia

Symposium. No. 156: Developments in Astrometry \& Their Impacts on Astrophysics and Geodynamics.

Symposium No. 158: Very High Angular Resolution Imaging.

Colloquium No. 140: Astronomy with Millimeter \& Submillimeter Wave Interferometers.

Advances in VLBI Techniques

Rogers, A. E. E., Rev. Radio Science, 1990-1992, p657, Pub. by URSI.

VLBI Technology

Proceedings of URSI/IAU Symposium on VLBI TECHNOLOGY -Progress and Future Observational Possibilities-, eds. T. Sasao et al., Terra Publishing Co., Japan.

\section{Recent Pulsar Discoveries}

Bailes, M. and Johnston, M., Rev. of Radio Science, 1990-1992, Pub. by URSI. 
RADIO ASTRONOMY

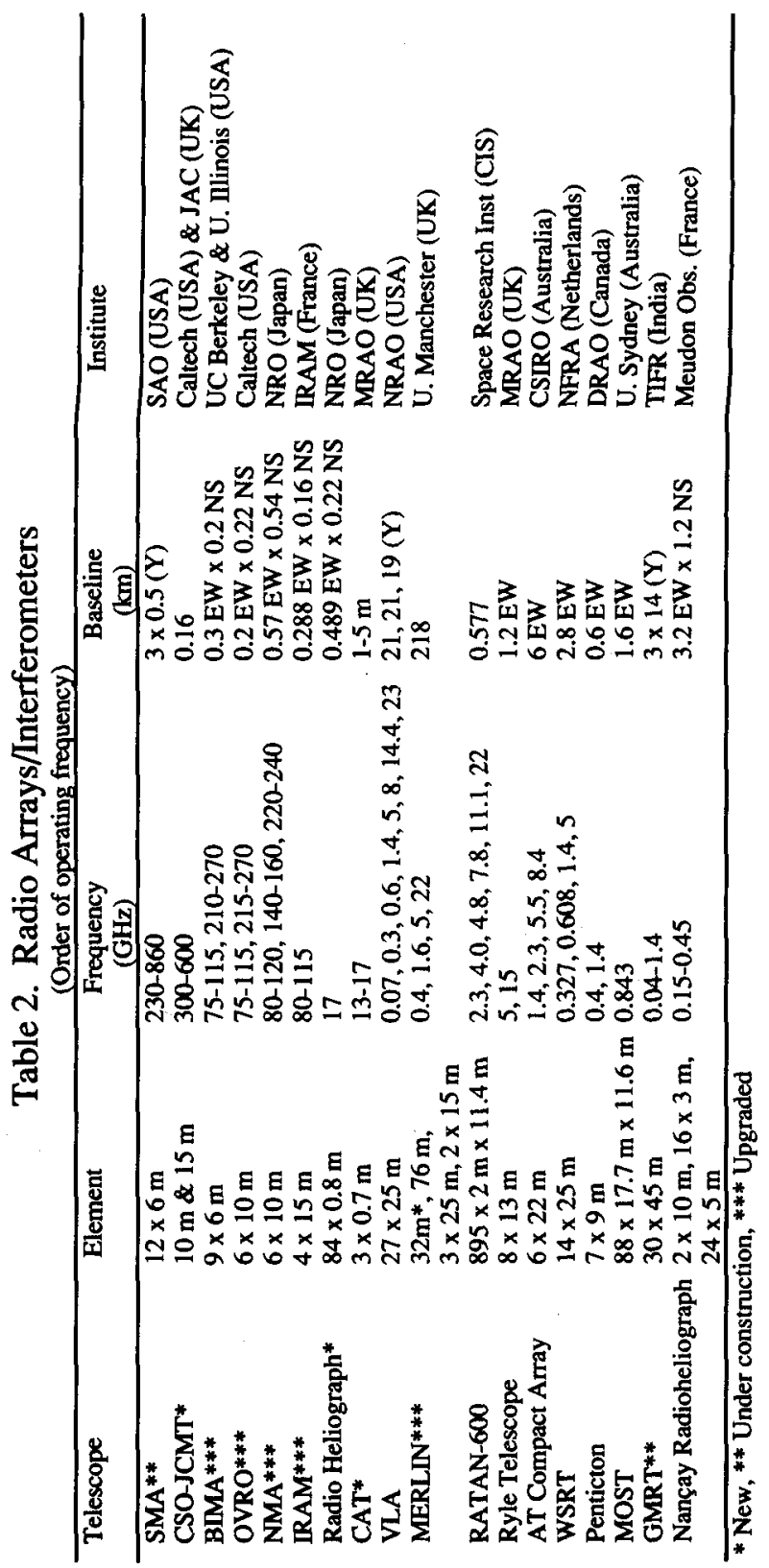

